\title{
Research on the designation of business model and bi-level optimization scheduling strategy of a VPP in energy market
}

\author{
Ming Zhang ${ }^{1}$, Mingxing Guo ${ }^{1}$, Bingqing Bai², Shufeng Guan ${ }^{2}$, Ran $\mathrm{Lyu}^{1 *}$, Su Wang ${ }^{1}$, Gaoxiu Xu ${ }^{2}$ \\ ${ }^{1}$ Economic Research Institute of State Grid Shanghai Electric Power Company, Shanghai 200233, China \\ ${ }^{2}$ School of Electronic Information and Electrical Engineering, Shanghai Jiao Tong University, Minhang District, Shanghai, 200240, \\ China
}

\begin{abstract}
With the rapid development of distributed energy resource, a more powerful market operation and energy management system is in great demand for a more precise scheduling strategy. And owing to the advantages in communication and control, virtual power plant (VPP) can provide solutions to these problems. In this paper, the business orientation and service plan are discussed first. Then the mathematical model of the settled market is established to figure out the scheduling strategy of participating in energy market. And a bi-level optimization model is built, with the upper level of maximizing the profits of VPP and lower level of clearing process of energy market. Finally, the scheduling scheme under typical scenarios is discussed in the example, and the ability of the VPP to adapt to the price change is analyzed. It can be seen that the VPP can adjust its own load and processing in the face of price fluctuation to achieve the maximum benefit.
\end{abstract}

\section{Introduction}

With the deepening of power organization reform and the promotion of power market construction, the obvious advantages of VPP in dealing with distributed energy gradually emerge. VPP can improve the power grid regulation ability, so as to promote renewable energy consumption [1-2]. Therefore, it is of great significance to study its business model and scheduling strategy.

In order to integrate the VPP into the current electricity market environment, designing a proper business model of VPP is crucial. Ref [3] introduces the construction of VPP in the United States, Australia and other countries, and makes a detailed analysis of the mechanism of peak load and frequency regulation service. VPP are usually connected to the power system from the low-voltage distribution network [4]. Ref [5] analyzes the market clearing strategy of distribution network with the participation of VPP considering various transaction types. Ref [6] analyzes the idea of distributed generation and controllable load participating in power market transaction, and discusses the influence of dispatching strategy. Based on the literature review of [7-9], this paper analyzes the mechanism of VPP participating in the virtual energy market.

On the basis of these studies, this paper considers the participation of VPP in energy market, designs the business model and mathematical model of VPP and the market in order to acquire the feasible scheduling strategy.

The main contribution is as follows:
- A business model of VPP is firstly designed. This paper mainly analyzes the market demand and the business orientation of VPP, and then the service plan that can be adopted by VPP is designed. Meanwhile the elements of the business mode are constructed qualitatively.

- On the basis of the proposed business model, considering the participation of VPP in energy market and natural gas market, the mathematical model of VPP scheduling process is established. Then the energy management strategy of VPP is analyzed in detail, and the feasibility of participating in energy market strategy is verified from the perspective of economic benefits of VPP's operation. 


\section{Business model of VPP}

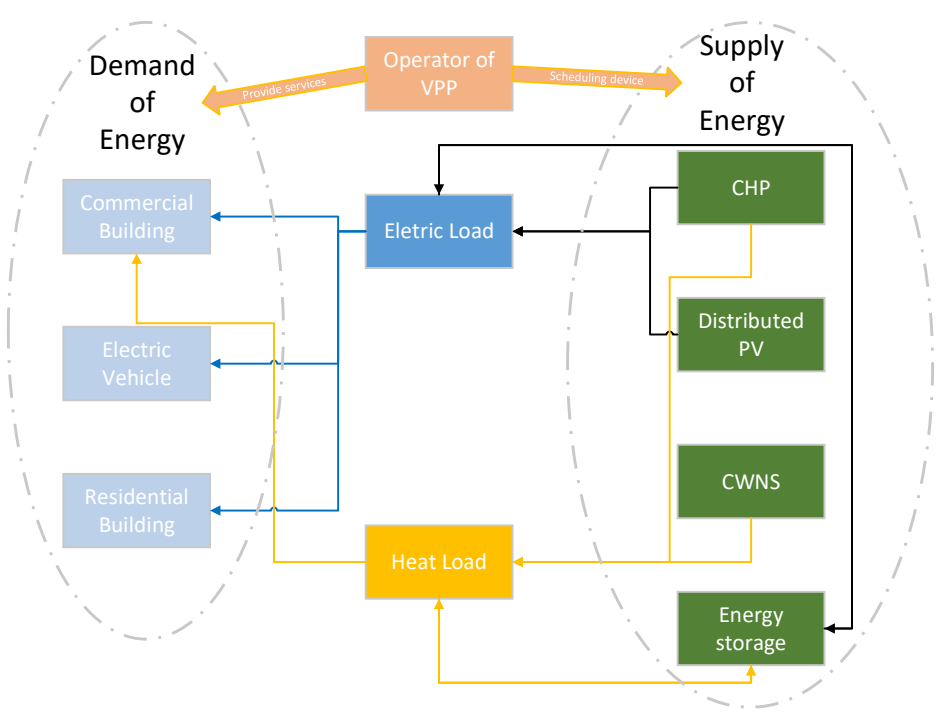

Figure 1. A typical structure of VPP

The structure of the VPP studied in this paper is shown in Figure 1, including all kinds of energy device, commercial buildings, residential buildings, and electric vehicles, among which the operator of VPP is the decision-making subject studied in this paper. The operator of VPP connects with users to provide electricity supply and heating services for users, controls the energy conversion and storage device and makes energy scheduling arrangements.

\subsection{Business Orientation}

Internally, the VPP has two functions: energy dispatching and economic operation. VPP needs to determine the scheduling scheme in the plant to maintain the real-time balance of energy supply and demand; Secondly, the VPP needs to ensure the normal economic operation in the area, formulate commercial packages of electricity and thermal energy, and reach energy supply contracts with users.

Externally, the VPP participates in the external power market as an electric energy user or generator and replaces the unit or user to conduct electricity transactions, obeying the management of the dispatch center such as the external power grid, and can make demand-side response to the price information. As a natural gas user, it purchases energy from the natural gas market to generate electricity; as an independent auxiliary service provider, it negotiates with the external power grid and signs a contract to participate in the auxiliary service market and obtains certain benefits.

\subsection{Service Contents}

- Basic energy services

VPPs provide users with energy package services, including power supply services, central heating services, and gas supply services for privately operated natural gas units and other natural gas loads. The VPP can calculate users' energy consumption habits, predict users' demand and energy purchase price, consider the current state of device in the area, and make an energy use scheme that conforms to the maximum economic benefits of the integrated energy system. The scheme includes the total consumption of various types of energy in a certain period of time and the distribution curve of various types of energy with respect to the period of energy consumption.

- Electrical auxiliary services

Peak shaving, frequency modulation services can be realized independently by internal electric energy storage device, or through the control of electric and heating loads in the area, combined with CHP units, distributed photovoltaic and thermal energy storage device. When the power supply of external grid is insufficient, it can provide active power support to the grid. When the external grid power supply is too large to be absorbed, the VPP absorbs with the help of user demand-side response management or electric thermal energy storage.

- Value-added services

The VPP provides energy management services. The VPP monitors and manages the electric heating supply through the intelligent terminal, masters the user's energy consumption data and optimizes the operation mode, and then uses the information device to guide the user's energy use plan and provide the user with energy management services. Meanwhile the VPP serves as an intermediate manager between the grid company and users to help the grid and users realize demand response management.

\section{Bi-level optimization model of VPP}

In this section, the mathematical model of VPP participating in energy market transaction is established. Firstly, the upper model of the VPP is established, and then the lower level model of external energy market is established to clear out the market with the goal of 
maximizing social welfare. Finally, with the help of KKT condition, the complete model is built.

\subsection{Upper layer model}

The VPP participates in the energy market on behalf of users, and considers the maximum utility of its own users and the lowest energy cost as a trading strategy to participate in the market. Therefore, the goal of the VPP is to maximize benefits, and the objective function can be described as

$$
\max \sum_{t}^{T}\left(U_{e, t} \times P_{e, t}+U_{h, t} \times P_{h, t}-\rho_{e s, t} \times d_{e s, t}-\rho_{g a s, t} \times d_{g a s, t}-O_{D G, t} \times P_{D G, t}\right)
$$

Where: the subscript $\mathrm{t}$ is the value of the variable at the corresponding time. $U_{e, t}$ and $U_{h, t}$ are user's electrical and heat load utility value, $P_{e, t}$ and $P_{h, t}$ the electrical load and heat load. $\rho_{e s, t}$ is the electricity price of the node where the VPP is located after the clearance of the dayahead electricity market, $\rho_{\text {gas }, t}$ is the gas price of the node where the VPP is located, $d_{e s, t}$ and $d_{\text {gas }, t}$ are the amount of electricity and gas that the VPP interacts with the energy market. $O_{D G, t}$ is the distributed power generation cost (except natural gas cost), $P_{D G, t}$ is the output.

The constraints considered include energy bus constraints, upper and lower power constraints of each device, energy storage device constraints, load constraints and VPP quotation constraints. Where the electric energy balance constraint is:

$$
\begin{gathered}
P_{e, t}=P_{e g, t}+P_{p v, t}+P_{e, t}^{G T}-P_{e s, t} \\
P_{e, t}^{G T}=h_{g a s} P_{g a s, t}^{G T} \eta_{G T}^{e}
\end{gathered}
$$

where: $P_{e, t}$ is the electric load required by the user, $P_{e g, t}$ is the interactive electric quantity with the grid, $P_{p v, t}$ is the output of photovoltaic power generation, $P_{e, t}^{G T}$ is the gas turbine power generation, $P_{e s, t}$ is the charging and discharging power of electric energy storage device, $h_{\text {gas }}$ is the calorific value of natural gas, $P_{g a s, t}^{G T}$ is the amount of natural gas consumed by the gas turbine, $\eta_{G T}^{e}$ is the power generation efficiency of the gas turbine.

\subsection{Lower layer model}

The VPP participates in a fully competitive day-ahead electricity market and gives a quotation according to market conditions. Other participants include generator sets and loads that declare the price or quantity of electricity according to their marginal utility. The electricity market clearing is carried out by an independent system operator and the goal of electricity market clearing is to minimize social cost. The objective function of power market model can be described as

$$
\min \left(\sum_{i}^{k}\left(O_{i}^{g} \times P_{i}^{g}\right)-\sum_{i}^{m}\left(U_{i}^{c} \times d_{i}^{c}\right)-J_{h} \times d_{e s}\right)
$$

where, $O_{i}^{g}$ is the generation cost of power-side generator set, $P_{i}^{g}$ is the planned output value of the generating set in the past day, $\mathrm{k}$ represents $\mathrm{k}$ generating sets in the regional power grid, In the area where the VPP is located, besides the VPP, there are $\mathrm{m}$ other fully competitive loads, $U_{i}^{c}$ is the electricity utility of the load and $d_{i}^{c}$ is the electricity consumption of the load. $J_{h}$ is the reported value of the VPP participating in the electricity market, $d_{e s}$ is the planned electricity purchase of the VPP.

The constraints considered include day-ahead market power balance constraint, generator output constraint, network security power flow constraint and node phase Angle constraint.

Among them, day-ahead market power balance constraint is:

$$
P_{t}^{g}-B_{b u s} \theta_{t}-d_{e s, t}-d_{c, t}=0: \lambda_{t}^{e}
$$

where, $P_{t}^{g}$ is the output of generating units at each node $\mathrm{t}, B_{b u s}$ is the grid network structure matrix, $\theta_{t}$ is the phase Angle vector of each node, $d_{e s, t}$ is the electricity load at the node where the VPP is located, $d_{c, t}$ is the user load, $\lambda_{t}^{e}$ is the dual variable of this constraint and the node electricity price of each node.

Similar to the power market, the natural gas market objective function can be described as:

$$
\min \left(\sum_{i}^{n}\left(O_{i}^{w} \times q_{i}^{w}\right)-\sum_{i}^{l}\left(U g_{i}^{c} \times d g_{i}^{c}\right)-J g \times d_{g a s}\right)
$$

Constraints also include natural gas power balance constraints, natural gas well output constraints, tie line constraints, other load constraints and network security flow constraints.

\section{Case studies}

\subsection{Basic Architecture of the System}

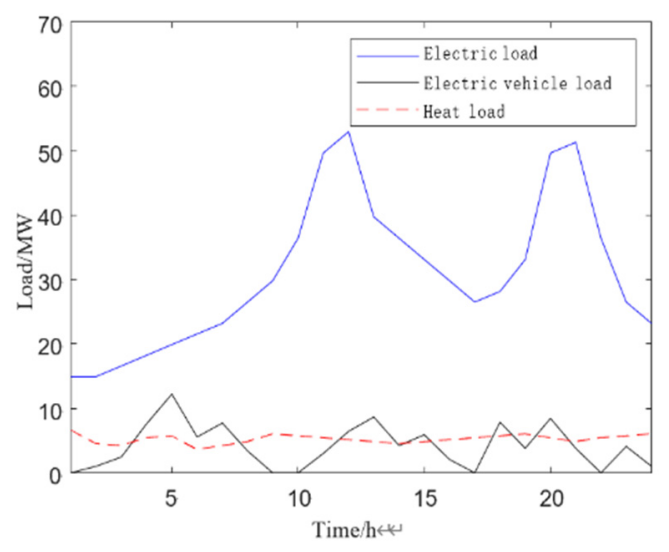

Figure 2. 24h typical load 


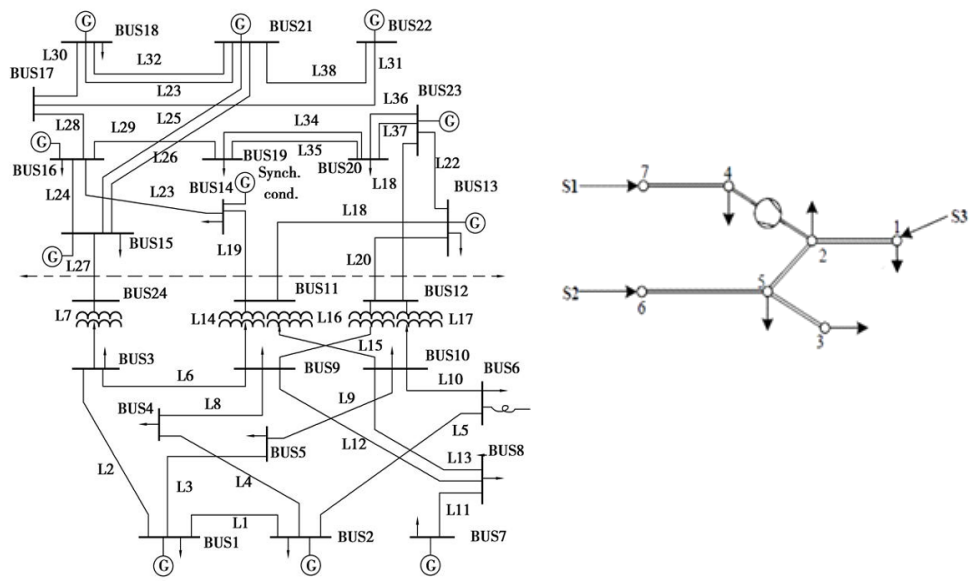

Figure 3. Network topology of VPP

In this section, the actual planning VPP is used to analyze the case to verify the validity of the model and analyze the strategy of the VPP. The device includes a 20MW CHP unit, a 40MW distributed photovoltaic unit, a $20 \mathrm{MWh}$ gas-fired boiler, a $10 \mathrm{WHh}$ electric energy storage thermal standby and a thermal energy storage device.

The load demand has electric heating load demand, and the electric load includes ordinary user load and electric vehicle load, as shown in Figure 2. The power system network structure used in this article is IEEE-

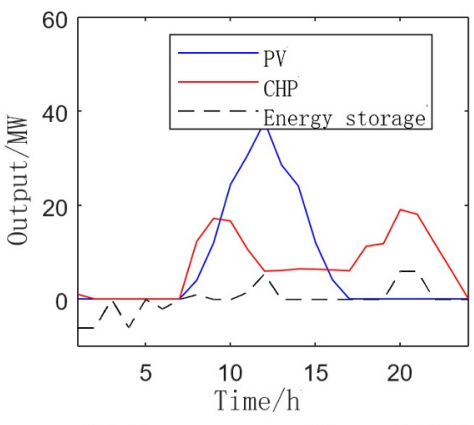

(a) Power generation of VPP

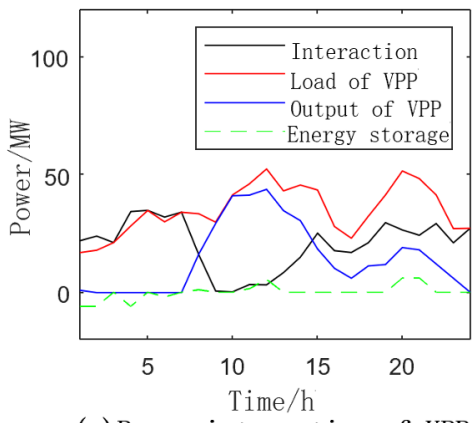

(c) Power interaction of VPP

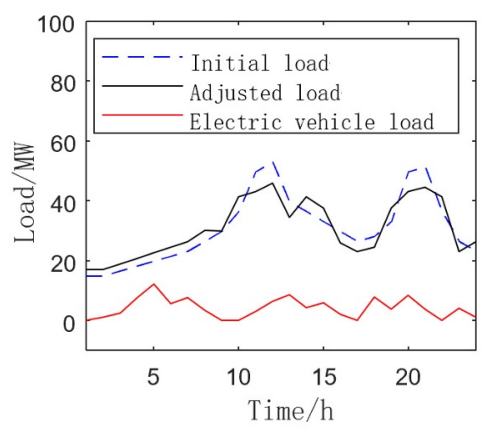

(b)Load of VPP

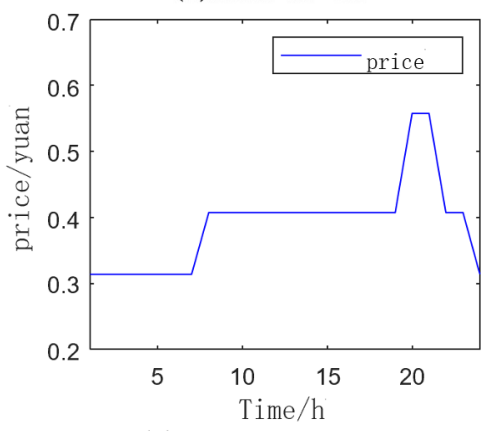

(d) Interaction price

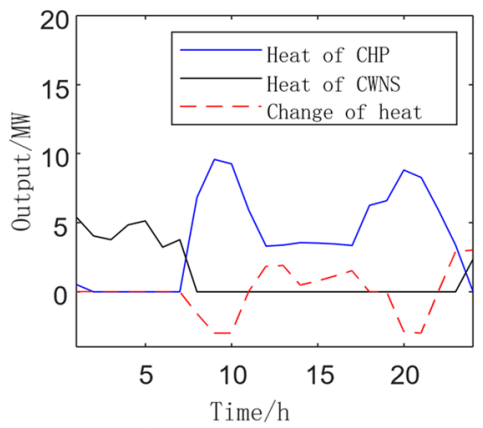

(a) Generated heat of VPP

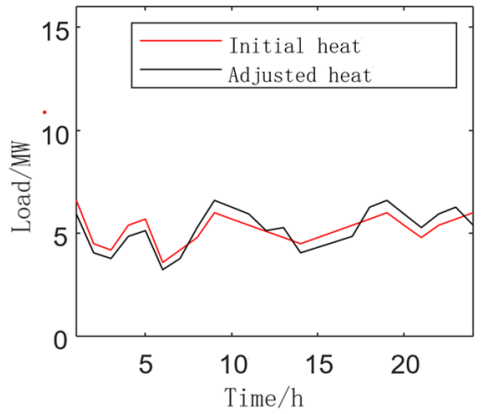

(b) Used heat of VPP
Figure 4. Electric energy marketoperation

Figure 4 shows the operation of the internal electric energy of the VPP in a typical scenario. The output of VPP increases with the price of electricity. It can be seen that in the $1 \mathrm{~h}-7 \mathrm{~h}$ period, because the clearing price in the day-ahead market is relatively low, electric energy is mainly purchased from the external market to meet the demand of electric load. During the period of $7 \mathrm{~h}-12 \mathrm{~h}$, the total load demand in the power market is moderate, and
Figure 5. Thermal energy market operation

the clearing price in the day-ahead market rises to a medium state. The VPP basically stops purchasing electricity from the outside after $9 \mathrm{~h}$ due to the increase of its own generating capacity. CHP unit generates electricity at a high rate in the $8 \mathrm{~h}-11 \mathrm{~h}$ period and begin to reduce their own output around $12 \mathrm{~h}$ of the peak of distributed photovoltaic power generation to ensure the balance of energy supply. Energy storage releases 
electric energy at the peak of electricity consumption, and the user side also starts to substantially shift load demand after $10 \mathrm{~h}$, reducing energy consumption costs. During the period from $19 \mathrm{~h}$ to $21 \mathrm{~h}$, the user load reaches the second peak, so VPP behaves the same way.

- Operation of thermal energy market

Figure 5 shows the thermal energy operation of the VPP under typical scenarios. It can be seen that the operation of the thermal energy system of the VPP is affected by the fluctuation of electricity prices. The thermal energy storage device and the CHP unit are in complementary working conditions. At the same time, the user side of the thermal load responds to the impact of electricity prices and shifts more of the thermal load to the time when the CHP unit supplies more heat, reducing energy costs through thermal-electric co-generation.

- Benefit analysis

It can be calculated from the operation of the VPP that in the current scenario, the user's energy utility is 531,400 yuan, the external energy purchase cost is 295,400 yuan, and the device operation and maintenance costs are 142,000 yuan. The VPP can obtain a profit of $94000 y u a n$ through energy transactions on this day. The return on the day was $21.5 \%$. It can be seen that the economic benefits of the VPP operation are very good under typical scenarios.

\section{Conclusion}

This article combines VPP's business model with the goal of maximizing the benefits of the VPP itself to construct a scheduling model for its participation in the energy market and provide an expandable solution for the future VPP operation, which mainly contains the following conclusions:

- The business model of VPP should be based on its own business orientation. It can be developed from the perspective of basic energy services, power auxiliary services and other value-added services.

- In the process of participating in the energy market, VPP can adjust its output of generator sets, energy storage device and controllable loads to adapt to the price changes of the electricity market and effectively improve the profit.

\section{Acknowledgments}

The authors gratefully acknowledge the support of Shanghai Science and Technology Plan Funded Project "Research and application for key technologies of public building VPP based on distributed resource aggregation control" (No. 20dz1206200).

\section{References}

1. CHEN, C., LI, N., ZHONG, P., \& ZENG, M. (2013). Review of virtual power plant technology abroad and enlightenment to China. Power System Technology, 37(8), 2258-2263.
2. Zhao, Q., Shen, Y., \& Li, M. (2015). Control and bidding strategy for virtual power plants with renewable generation and inelastic demand in electricity markets. IEEE Transactions on Sustainable Energy, 7(2), 562-575.

3. LI Jiamei., AI Qian., \& YIN Shuangrui.(). Market Mechanism and Foreign Experience of Virtual Power Plant Participating in Peak-regulation and Frequency-regulation. Proceedings of the CSEE (), doi:10.13334/j.0258-8013.pcsee.202152.

4. Zangeneh, A., Shayegan-Rad, A., \& Nazari, F. (2018). Multi-leader-follower game theory for modelling interaction between virtual power plants and distribution company. IET Generation, Transmission \& Distribution, 12(21), 5747-5752.

5. Yi, Z., Xu, Y., Gu, W., \& Wu, W. (2019). A multitime-scale economic scheduling strategy for virtual power plant based on deferrable loads aggregation and disaggregation. IEEE Transactions on Sustainable Energy, 11(3), 1332-1346.

6. Palma-Behnke, R., Vargas, L. S., \& Jofré, A. (2005). A distribution company energy acquisition market model with integration of distributed generation and load curtailment options. IEEE Transactions on Power Systems, 20 (4), 1718-1727.

7. Mashhour, E., \& Moghaddas-Tafreshi, S. M. (2010). Bidding strategy of virtual power plant for participating in energy and spinning reserve markets-Part I: Problem formulation. IEEE Transactions on Power Systems, 26 (2), 949-956.

8. Heredia, F. J., Rider, M. J., \& Corchero, C. (2010). Optimal bidding strategies for thermal and generic programming units in the day-ahead electricity market. IEEE Transactions on Power Systems, 25 (3), 1504-1518.

9. TIAN Liting., CHENG Lin., GUO Jianbo., WANG Xuanyuan., YUN Qiuchen., \& GAO Wenzhong. (2020). A Review on the Study of Management and Interaction Mechanism for Distributed Energy inVirtual Power Plants. Power System Technology. Power System Technology, 44 (6), 2097-2108. 